\title{
ISOMETRIES HOMOTOPIC TO THE IDENTITY
}

\author{
DOUGLAS A. NORRIS
}

(Communicated by David G. Ebin)

\begin{abstract}
The types of surfaces which admit nontrivial isometries homotopic to the identity are classified up to diffeomorphism. In dimension three this is done for complete manifolds of constant negative curvature. Three-dimensional visibility manifolds that admit nontrivial isometries homotopic to the identity are shown to be diffeomorphic to a product $L \times R^{1}$.
\end{abstract}

\section{INTRODUCTION}

A theorem of Eberlein [4] states that if a surface, that is, a two-dimensional connected Riemannian manifold, is complete, has nonpositive Gaussian curvature, and is not homeomorphic to a plane, cylinder, Möebius band, torus or Klein bottle, then its isometry group is discrete. It is an effort, in dimension two, to remove the condition of compactness from a classical theorem of Bochner [3]. Bochner proved that a compact $n$-dimensional manifold with negative Ricci tensor has a discrete and therefore finite isometry group. A stronger conclusion to Eberlein's theorem would be that any isometry of the surface homotopic to the identity must be the identity. This is precisely the way in which Frankel [6] modified Bochner's theorem, although he needed to make the additional assumption that the manifold must have nonpositive sectional curvature.

The purpose of this paper is to improve Eberlein's theorem for surfaces in a similar way and then to obtain analogous results in dimension three. It will be shown that without curvature or completeness restrictions, up to diffeomorphism, there are seven types of surfaces which admit nontrivial isometries homotopic to the identity. Three-dimensional visibility manifolds which admit nontrivial isometries homotopic to the identity will be shown to be diffeomorphic to a product $L \times R^{1}$ where $L$ is a submanifold. The additional condition of constant negative curvature will allow such a three-dimensional manifold to be classified, up to diffeomorphism, as one of five types.

\section{CONVEX FUNCTIONS AND VISIBILITY MANIFOLDS}

The results of this paper depend on the following properties of convex functions and visibility manifolds (refer to [1, pp. 77-85], [2], and [5]).

Received by the editors September 28, 1986 and, in revised form, March 1, 1988.

1980 Mathematics Subject Classification (1985 Revision). Primary 53C20. 
A continuous function $g: M \rightarrow R^{1}$ on a complete Riemannian manifold $M$ is convex if for every geodesic segment $\gamma: I \rightarrow M$ from any $m$ to any $n$ and for every $t \in I$, we have $g(\gamma(t)) \leq(1-t) g(m)+t g(n)$. A subset $N$ of $M$ is called convex, if for $m, n \in N$ there is (up to parametrization) a unique shortest geodesic from $m$ to $n$ in $M$ and this geodesic is contained in $N$.

A Hadamard manifold is a complete, connected, simply connected Riemannian manifold of dimension $n \geq 2$ having nonpositive sectional curvature. Such manifolds are diffeomorphic to $R^{n}$. The complete manifolds $M$ of dimension $n \geq 2$ having nonpositive sectional curvature are precisely the quotient manifolds $\widetilde{M} / \Gamma$ where the Riemannian covering $\widetilde{M}$ is a Hadamard manifold and $\Gamma$ is a freely acting properly discontinuous group of isometries of $\widetilde{M}$. The covering manifold $\widetilde{M}$ can be compactified by the addition of points at infinity. Let $\widetilde{M}(\infty)$ denote the set of points of infinity of $\widetilde{M}$ where a point of infinity is an asymptote class of unit speed geodesics of $\widetilde{M}$. A natural topology can be defined on $\widetilde{M} \cup \widetilde{M}(\infty)$ such that $\widetilde{M}(\infty)$ is homeomorphic to $S^{n-1}$. If $\tilde{\sigma}$ is an isometry of $\widetilde{M}$ then this map can be extended to a homeomorphism of $\widetilde{M} \cup \widetilde{M}(\infty)$ which will also be denoted by $\tilde{\sigma}$. The set of accumulation points of an orbit $\Gamma(q)=\{\gamma(q): \gamma \in \Gamma\}$ in $\widetilde{M}(\infty)$ is independent of the choice of $q \in \widetilde{M}(\infty)$ and is called the limit set $L(\Gamma)$ of $\Gamma$.

The isometries of a Hadamard manifold $\widetilde{M}$ can be classified using the displacement function. If $\tilde{\sigma}$ is an isometry on $\widetilde{M}$, the convex function $d_{\tilde{\sigma}}(x)=$ $d(x, \tilde{\sigma}(x))$, where $d($,$) denotes the distance on \widetilde{M}$, is called the displacement function of $\tilde{\sigma}$. An isometry $\tilde{\sigma}$ is called elliptic, hyperbolic or parabolic if $d_{\tilde{\sigma}}$ has, respectively, zero minimum, positive minimum or no minimum in $\widetilde{M}$.

Let $\sigma$ be an isometry homotopic to the identity of the complete Riemannian manifold of nonpositive sectional curvature $M=\widetilde{M} / \Gamma$. Let the homotopy be denoted by $h_{t}: M \rightarrow M$ where $h_{0}$ is the identity transformation of $M$ and $h_{1}=\sigma$, and define $\tilde{h}_{t}: \widetilde{M} \rightarrow \widetilde{M}$ to be the unique lift of $h_{t}$ to $\widetilde{M}$ such that $\tilde{h}_{0}$ is the identity mapping $[8, \mathrm{pp} .57-58]$. Then $\tilde{\sigma}=\tilde{h}_{1}$ commutes with every deck transformation. Define $f_{\sigma}$ to be the function on $M$ where $f_{\sigma}(m)$ is the length of the unique geodesic segment from $m$ to $\sigma(m)$ in the homotopy class of the curve $t \rightarrow h_{t}(m)$. Given any point $\tilde{m} \in p^{-1}(m)$ where $p$ is the covering map, the geodesic segment lifts to a geodesic segment in $\widetilde{M}$ starting at $\tilde{m}$ and its length is $d_{\tilde{\sigma}}(\tilde{m})$. Since $\tilde{\sigma}$ commutes with every deck transformation, it follows that $d_{\tilde{\sigma}}(\tilde{m})=f_{\sigma}(m)$ for every $\tilde{m} \in p^{-1}(m)$. Therefore, $f_{\sigma}$ is convex and the level sets of $d_{\tilde{\sigma}}$ project to level sets of $f_{\sigma}$ via $p$. The isometry $\sigma$ will be called elliptic, hyperbolic or parabolic if $f_{\sigma}$ has, respectively, zero minimum, positive minimum, or no minimum in $M$. This causes the isometry $\tilde{\sigma}$ to be given the same classification name as $\sigma$. If $f_{\sigma}$ assumes a minimum on $M$ then let $C_{\sigma}$ denote the minimum set. This minimum set $C_{\sigma}$ is the projection of the minimum set of $d_{\tilde{\sigma}}$ on $\widetilde{M}$ which is analogously denoted $C_{\tilde{\sigma}}$. Both $C_{\sigma}$ and $C_{\tilde{\sigma}}$ are closed convex sets. 
A manifold $M=\widetilde{M} / \Gamma$ where $\widetilde{M}$ is a Hadamard manifold is called a visibility manifold if any two points in $\widetilde{M}(\infty)$ can be joined by a geodesic. The class of visibility manifolds includes all complete manifolds of curvature $K \leq c<0$. An isometry $\sigma$ on $M$ can be characterized by the number of points $F(\sigma)$ that its lift $\tilde{\sigma}$ fixes in $\widetilde{M}(\infty)$. Since $M$ is a visibility manifold $F(\sigma)$ is either $0,1,2$ or $\infty$.

(i) If $\tilde{\sigma}$ is elliptic, then $C_{\tilde{\sigma}}$ is a complete, closed, connected, totally geodesic submanifold without boundary, and if $\tilde{\sigma}$ is nontrivial then $\operatorname{dim} C_{\tilde{\sigma}}<\operatorname{dim} M$. The isometry $\tilde{\sigma}$ fixes either 0 points in $\widetilde{M}(\infty)$ when $C_{\tilde{\sigma}}$ is an isolated fixed point, 2 points or an infinite number of points.

(ii) If $\tilde{\sigma}$ is hyperbolic, then $\tilde{\sigma}$ fixes exactly 2 points in $\widetilde{M}(\infty)$.

(iii) If $\tilde{\sigma}$ is parabolic, then $\tilde{\sigma}$ fixes exactly 1 point in $\widetilde{M}(\infty)$ and $\tilde{\sigma}$ leaves the horospheres at that point invariant.

A visibility manifold $M=\widetilde{M} / \Gamma$ can be classified as parabolic, axial, or fuchsian. If $L(\Gamma)$ contains exactly one point then $M$ is called parabolic and if $L(\Gamma)$ contains exactly two points then $M$ is called axial. A visibility manifold that is neither parabolic nor axial is said to be fuchsian.

\section{Classification of EXCEPTIONAL MANIFOldS}

Exceptional manifolds are those which admit nontrivial isometries homotopic to the identity. The primary strategy is to show that the restrictions placed upon the manifold cause it to be either parabolic or axial.

Proposition. Let $M$ be a visibility manifold. If $M$ admits a nontrivial isometry $\sigma$ homotopic to the identity such that $F(\sigma)$ is finite then $M$ is diffeomorphic to a product $L \times R^{1}$ unless $M$ is a Möebius band.

Proof. (Refer to [2], [5] and [7].) If the manifold $M$ is simply connected then the group of deck transformations $\Gamma$ of $M=\widetilde{M} / \Gamma$ is trivial and $M$ is diffeomorphic to $R^{n}$ and therefore to $R^{n-1} \times R^{1}$. Assume that $\Gamma$ is nontrivial. The limit set $L(\Gamma)$ must be nonempty since $\Gamma$ is an infinite group that acts freely and properly discontinuously on $\widetilde{M}$. If $x \in L(\Gamma)$, then choose a sequence $\left\{\gamma_{n}\right\}$ of isometries of $\Gamma$ such that $\gamma_{n}(q) \rightarrow x$ as $n \rightarrow \infty$ for any point $q \in \widetilde{M}$. Since $\tilde{\sigma}$ commutes with each element of $\Gamma, \tilde{\sigma}(x)=\lim _{n \rightarrow \infty} \tilde{\sigma} \gamma_{n}(q)=$ $\lim _{n \rightarrow \infty} \gamma_{n} \tilde{\sigma}(q)=x$. Thus $\tilde{\sigma}$ fixes each point of $L(\Gamma)$ and the cardinality of $L(\Gamma)$ is at most $F(\sigma) \leq 2$. Therefore, $L(\Gamma)$ contains either one or two points.

If $L(\Gamma)$ contains one point $x$, then $M$ is a parabolic manifold and the $C^{2}$ Busemann function at $x$ induces a $C^{2}$ convex function $f$ on $M$ which has no minimum. The manifold $M$ is therefore diffeomorphic to a product $L \times R^{1}$ where $L$ is a level surface of $f$, the projection of a horosphere in $\widetilde{M}$ at $x$.

If $L(\Gamma)$ contains two points, then $\widetilde{M} / \Gamma$ is an axial manifold. An axial manifold is a smooth vector bundle over a circle and is therefore diffeomorphic to either $S^{1} \times R^{n-1}$ or $B \times R^{n-2}$ where $B$ is the Möebius band. 
Corollary. Let $M$ be a complete $n$-dim manifold of constant negative curvature such that $n \geq 3$. If $M$ admits a nontrivial isometry $\sigma$ homotopic to the identity such that $F(\sigma)$ is finite then $M$ is diffeomorphic to $L \times R^{1}$ where $L$ is an $(n-1)$-dim submanifold that admits a complete flat metric.

Proof. It follows from the proof of the proposition that if $M$ is not simply connected then it is either parabolic or axial. If $M$ is simply connected then it is diffeomorphic to $R^{n-1} \times R^{1}$, and $R^{n-1}$ admits a flat metric. A parabolic manifold is diffeomorphic to $L \times R^{1}$ where $L$ is the projection of a horosphere. In this case, $L$ is flat since $M$ has constant curvature and horospheres are flat in the induced metric. An axial manifold is diffeomorphic to either $S^{1} \times R^{n-1}$ or $B \times R^{n-2}$, and $S^{1} \times R^{n-2}$ and $B \times R^{n-3}$ both admit a flat metric.

Theorem 1. If a surface $M$ is not diffeomorphic to a plane, cylinder, Möebius band, torus, Klein bottle, sphere or projective plane, then the only isometry of $M$ homotopic to the identity is the identity.

Proof. Let $\phi$ be a nontrivial isometry of a surface $M$ which is homotopic to the identity. The surface $M$ is conformally equivalent to a complete surface of constant Gauss curvature [ 9 and 10]. Therefore $M$ is conformally equivalent to a space form; either $S^{2} / \Gamma, R^{2} / \Gamma$, or $H^{2} / \Gamma$ where $H^{2}$ is the hyperbolic plane. Up to isometry $S^{2} / \Gamma$ can only be either a sphere or a projective plane while $R^{2} / \Gamma$ can only be the plane, a cylinder, a torus, a Móebius band or a Klein bottle. These are all exceptional cases in the statement of the theorem. So we can assume there exists a conformal diffeomorphism $\rho: M \rightarrow H^{2} / \Gamma$. The mapping $\sigma=\rho \phi \rho^{-1}$ and the lift $\tilde{\sigma}$ are nontrivial conformal diffeomorphisms homotopic to the identity on $H^{2} / \Gamma$ and $H^{2}$, respectively. The conformal diffeomorphisms of $H^{2}$ are known to be precisely the isometries of $H^{2}$ and it follows that $\tilde{\sigma}$ and therefore $\sigma$ are isometries.

If $\sigma$ is nonelliptic then the proposition applies and $H^{2} / \Gamma$ is diffeomorphic to either the Möebius band or to $L \times R^{1}$ which in the latter case can only be the plane or the cylinder. If $\sigma$ is an elliptic isometry, then the lift $\tilde{\sigma}$ is also a nontrivial elliptic isometry and $\operatorname{dim} C_{\tilde{\sigma}}<\operatorname{dim} H^{2}=2$. Thus $\operatorname{dim} C_{\sigma} \leq 1$, which implies that $F(\sigma)$ is at most 2, and the proposition again applies.

Theorem 2. Let $M$ be a three-dimensional visibility manifold. If $M$ is not diffeomorphic to a product $L \times R^{1}$ then the only isometry of $M$ homotopic to the identity is the identity.

Proof. If $\sigma$ is a nonelliptic isometry which is nontrivial then the result follows from the proposition. Let $\sigma$ be a nontrivial elliptic isometry homotopic to the identity. Assume that $\operatorname{dim} C_{\tilde{\sigma}}=2$. Since $\tilde{\sigma}$ is homotopic to the identity, and since the manifold is three dimensional, the induced mapping $\tilde{\sigma}_{*}$ of the tangent bundle must fix the tangent space at each point of the fixed point set $C_{\tilde{\sigma}}$. This would imply that $\tilde{\sigma}$ is the identity mapping. Therefore, if the isometry $\sigma$ is elliptic then $\operatorname{dim} C_{\sigma}=\operatorname{dim} C_{\tilde{\sigma}} \leq 1$ and the theorem is a consequence of the proposition. 
In the following corollary the symbol $T$ represents the torus, $B$ the Möebius band, and $K$ the Klein bottle.

Corollary. Let $M$ be a complete connected three-dimensional Riemannian manifold of constant negative curvature. If $M$ is not diffeomorphic to $R^{3}, S^{1} \times R^{2}$, $T \times R^{1}, B \times R^{1}$, or $K \times R^{1}$ then the only isometry of $M$ homotopic to the identity is the identity.

Proof. If $\sigma$ is a nontrivial isometry homotopic to the identity then Theorem 2 and the corollary to the proposition imply that $M$ is a product $L \times R^{1}$ where $L$ is a two-dimensional manifold that admits a complete flat metric. Up to isometry, the complete, flat, two-dimensional manifolds are the plane, cylinder, torus, Möebius band, and Klein bottle.

The exceptional types of manifolds in Theorem 1 and in the above corollary are true exceptions. That is, there exist manifolds of each exceptional type which admit nontrivial isometries homotopic to the identity.

\section{REFERENCES}

1. Werner Ballman, Mikhael Gromov and Viktor Schroeder, Manifolds of nonpositive curvature, Birkhäuser, Boston, 1985.

2. R. Bishop and B. O'Neill, Manifolds of negative curvature, Trans. Amer. Math. Soc. 145 (1969), 1-49.

3. S. Bochner, Vector fields and Ricci curvature, Bull. Amer. Math. Soc. 52 (1946), 776-797.

4. P. Eberlein, Surfaces of nonpositive curvature, Mem. Amer. Math. Soc. No. 218 (1979).

5. P. Eberlein and B. O'Neill, Visibility manifolds, Pacific J. Math. 46 (1973), 45-110.

6. T. Frankel, On theorems of Hurwitz and Bochner, J. Math. Mech. 15 (1966), 373-377.

7. E. Heintze and H. C. Im Hof, Geometry of horospheres, J. Differential Geom. 12 (1977), 481-491.

8. S. Kobayashi, Transformation groups in differential geometry, Springer-Verlag, New York, 1972.

9. G. Springer, Introduction to Riemann surfaces, Addison-Wesley, Reading, Massachusetts, 1957.

10. J. A. Wolf, Spaces of constant curvature, McGraw-Hill, New York, 1967.

Department of Mathematics, John Carroll University, University Heights, Ohio 44118 